\title{
Editorial
}

\section{The most difficult task}

\section{Giovanni Lodi*}

Dipartimento di Scienze Biomediche, Chirurgiche e Odontoiatriche, Università degli Studi di Milano, Milano, Italy

Taking decisions on the management of a patient affected by a leukoplakia is probably one of the most difficult task in oral medicine practice.

In 1967, Jerzy Einhorn and Jan Wersall presenting their cohort of 782 patients, stated that "there is no evidence that the incidence of oral carcinoma can be diminished by surgical removal of the leukoplakia" [1]. Fifty years later, here we are again. We, the oral medicine specialists and clinical researchers, have not been able to plan and conduct even a single randomized controlled trial (RCT) to test the effectiveness of surgery in the treatment of patients affected by oral leukoplakia. This must be a great criticism to our community, although it is personal experience that funding bodies have shown little interest in supporting clinical research on a disorder affecting hundreds of thousands individuals around the world and likely to be responsible for a significant proportion of cancer of the mouth, a neoplasm still killing half of affected patients.

In absence of RCTs, evidence on surgical effectiveness in reducing incidence of cancer in patients with oral leukoplakia must be searched in observational studies [2]. And although most of them are small with short follow up, retrospective in nature and biased, i.e. unable to provide sound (and even notso-sound) evidence, the results of some of the best did not show significant benefits of surgical treatment [3-5].

For non-surgical treatment, the state of the art is just apparently better. In fact, as presented by a recent Cochrane review, although 14 RCTs testing a number of medical and complementary interventions are available, their overall quality is low or very low, and their findings uncertain [6]. Noteworthy, just five of them included cancer incidence among outcomes, only three of which provided usable data. The conclusion was that currently there is no evidence that any treatment is effective for preventing the development of oral cancer in subjects affected by oral leukoplakia.

Since we do not have any evidence for a treatment that works, a "wait and see" approach might be the most judicious choice. Many colleagues seem uncomfortable with it, equating

\footnotetext{
*Correspondence: giovanni.lodi@unimi.it
}

it with "doing nothing". This is absolutely incorrect, since "wait and see" needs an active attitude of both clinician and patient, to get involved in a strict program of surveillance, with frequent visits and biopsies, aimed to make a diagnosis of cancer as early as possible, thus providing the best possible prognosis.

A great advantage of "wait and see" is to limit overtreatment at minimum. It must be borne in mind that treatment of leukoplakia is prophylactic. In fact, the target is not the lesion itself, which does not represents a medical problem, but the prevention of cancer. Since we are not able to identify the relatively few who will develop cancer, or to exclude those that will never do it, we treat all patients with leukoplakia, the vast majority of which uselessly.

Another advantage is safety. A treatment that is useless in nine time out of ten should be, at least, absolutely safe. Something that is not true for a number of medical treatment proposed [6], and probably for surgery, particularly for large lesions (which, by the way, are those at higher risk) $[4,5,7]$.

Finally, "wait and see" must be considered indispensable, even in subjects who underwent treatments of any kind, including surgical removal, since none of the proposed treatments can eliminate, or even diminish, the risk of cancer, thus making a strict monitoring of the mouth of patients with leukoplakia an essential part of their management.

Thus, we can conclude, as usual, that a lot of clinical research is needed, but we must also add that in the meantime, we cannot but keep our patients with oral leukoplakia under strict surveillance.

\section{References}

1. Einhorn J, Wersall J. Incidence of oral carcinoma in patients with leukoplakia of the oral mucosa. Cancer 1967;20:2189-2193.

2. Warnakulasuriya $S$, Ariyawardana A. Malignant transformation of oral leukoplakia: a systematic review of observational studies. J Oral Pathol Med 2016;45:155-166.

3. Arduino PG, Surace A, Carbone M, Elia A, Massolini G, Gandolfo S, Broccoletti R. Outcome of oral dysplasia: a retrospective hospitalbased study of 207 patients with a long follow-up. J Oral Pathol Med. 2009;38:540-544. 
4. Brouns E, Baart J, Karagozoglu Kh, Aartman I, Bloemena E, van der Waal I. Malignant transformation of oral leukoplakia in a welldefined cohort of 144 patients. Oral Dis 2014;20:e19-e24.

5. Holmstrup P, Vedtofte P, Reibel J, Stoltze K. Long-term treatment outcome of oral premalignant lesions. Oral Oncol 2006;42: 461-474.
6. Lodi G, Franchini R, Warnakulasuriya S, Varoni EM, Sardella A, Kerr AR, Carrassi A, MacDonald LC, Worthington HV. Interventions for treating oral leukoplakia to prevent oral cancer. Cochrane Database Syst Rev 2016;29;CD001829.

7. Napier SS, Cowan CG, Gregg TA, Stevenson M, Lamey PJ, Toner PG. Potentially malignant oral lesions in Northern Ireland: size (extent) matters. Oral Dis 2003;9:129-137. 